Richard F. Bass

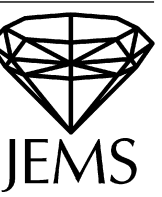

\title{
A stability theorem for elliptic Harnack inequalities
}

Received July 11, 2011 and in revised form October 19, 2011

Abstract. We prove a stability theorem for the elliptic Harnack inequality: if two weighted graphs are equivalent, then the elliptic Harnack inequality holds for harmonic functions with respect to one of the graphs if and only if it holds for harmonic functions with respect to the other graph. As part of the proof, we give a characterization of the elliptic Harnack inequality.

\section{Introduction}

A justly famous theorem of Moser [12] says that if $\mathcal{L}$ is the uniformly elliptic operator in divergence form given by

$$
\mathcal{L} f(x)=\sum_{i, j=1}^{d} \frac{\partial}{\partial x_{i}}\left(a_{i j}(\cdot) \frac{\partial f}{\partial x_{j}}(\cdot)\right)(x)
$$

acting on functions on $\mathbb{R}^{d}$, where the $a_{i j}$ are also bounded and measurable, then an elliptic Harnack inequality (EHI) holds for functions that are non-negative and harmonic with respect to $\mathcal{L}$ in a domain. This is one of the more important theorems in the study of elliptic and parabolic partial differential equations, and is used, for example, in deriving a priori regularity results for harmonic functions and for heat kernels.

The operator $\mathcal{L}$ is associated with the Dirichlet form

$$
\mathcal{E}_{\mathcal{L}}(f, f)=\int_{\mathbb{R}^{d}} \sum_{i, j=1}^{d} a_{i j}(x) \frac{\partial f}{\partial x_{i}}(x) \frac{\partial f}{\partial x_{j}}(x) d x .
$$

If the $a_{i j}$ are bounded and the matrices $a(x)=\left(a_{i j}(x)\right)$ are uniformly positive definite, then $\mathcal{E}_{\mathcal{L}}$ is comparable to $\mathcal{E}_{\Delta}$, where

$$
\mathcal{E}_{\Delta}(f, f)=\int_{\mathbb{R}^{d}}|\nabla f(x)|^{2} d x,
$$

which is the Dirichlet form corresponding to the Laplacian. Thus one could rephrase Moser's theorem as saying that whenever the Dirichlet form corresponding to an opera-

R. F. Bass: Department of Mathematics, University of Connecticut, Storrs, CT 06269-3009, USA; e-mail: bass@math.uconn.edu

Mathematics Subject Classification (2010): Primary 31B05; Secondary 31E05, 60J27 
tor $\mathcal{L}$ is comparable to the Dirichlet form corresponding to the Laplacian, then the EHI holds for non-negative functions that are harmonic with respect to $\mathcal{L}$ in a domain.

We can view Moser's theorem as a stability theorem for the EHI. The purpose of this paper is to generalize this stability property to very general state spaces. We show that provided some mild regularity holds, then whenever two Dirichlet forms $\mathcal{E}_{1}$ and $\mathcal{E}_{2}$ are comparable with corresponding operators $\mathcal{L}_{1}$ and $\mathcal{L}_{2}$, the EHI holds for non-negative harmonic functions with respect to $\mathcal{L}_{1}$ if and only if the EHI holds for non-negative harmonic functions with respect to $\mathcal{L}_{2}$.

We also provide a characterization of the EHI. Provided the regularity holds, this characterization can be considered as necessary and sufficient conditions for the EHI.

It is interesting to compare the EHI with the parabolic Harnack inequality (PHI). The PHI, first proved by Moser in [13] (see [7] for a very different proof), is a Harnack inequality for non-negative solutions to

$$
\frac{\partial u}{\partial t}(x, t)=\mathcal{L} u(x, t)
$$

in a domain. Necessary and sufficient conditions are known for the PHI in quite general state spaces. If the state space is regular enough to have a large class of nice cut-off functions, then Grigor'yan [9] and Saloff-Coste [14] independently proved that the PHI holds if and only if both volume doubling and a Poincaré inequality hold. This was extended to the case where such nice cut-off functions need not exist in [3] and [4]. The latter papers allow state spaces that have fractal structure or that have large numbers of obstructions.

If the PHI holds, then the EHI holds; this is quite easy to see. The converse is false. In [2] an example was given where EHI holds, but the PHI in the usual form does not (that is, with scaling factor $r^{2}$ ). Delmotte [6] constructed an example where the EHI holds, but volume doubling does not, and consequently the PHI cannot hold in any form. See [10] for more on the relationship between the EHI and PHI. It has been an open problem for quite some time to find a characterization of the EHI comparable to the one for the PHI.

In this paper we primarily look at infinite graphs rather than continuous state spaces. All the key ideas are present in the infinite graph case and we avoid some unpleasant technicalities. It is straightforward to extend our results to metric measure Dirichlet spaces in a manner very similar to how [4] extended [3]: see Section 7.

We consider infinite graphs where between any two adjacent vertices $x$ and $y$ there is given a conductance $C_{x y}$. If $x$ and $y$ are adjacent, we write $x \sim y$. Setting

$$
\mu_{x}=\sum_{z \sim x} C_{x z}
$$

we can construct a continuous time nearest neighbor Markov chain $X$ with the graph as the state space. When $X$ is at $x$, it waits an independent exponential length of time with parameter $\mu_{x}$ and then jumps to an adjacent vertex. It chooses a neighboring vertex $y$ with probability $C_{x y} / \mu_{x}$. We write $\mathcal{L}$ for the infinitesimal generator of $X$. A function $h$ is harmonic with respect to $\mathcal{L}$ in a domain $D$ if

$$
h(x)=\sum_{y \sim x} h(y) C_{x y}, \quad x \in D .
$$


Let $B(x, r)$ denote the ball of radius $r$ about $x$. The elliptic Harnack inequality states that there exists a constant $c$ not depending on $x_{0}$ or $r$ such that if $h$ is non-negative and harmonic in $B\left(x_{0}, 2 r\right)$, then

$$
h(x) \leq \operatorname{ch}(y), \quad x, y \in B\left(x_{0}, r\right) .
$$

We do require some mild regularity. For example, one of our assumptions is that volume doubling holds. Whereas the PHI implies volume doubling, the example of Delmotte [6] shows that the EHI can hold even though volume doubling does not. Since every known approach to proving an EHI uses volume doubling in an essential way, the problem of finding necessary and sufficient conditions for the EHI to hold without assuming any regularity looks very hard.

For most of this paper we consider the case where the process $X$ is transient, that is, $d\left(X_{t}, x\right) \rightarrow \infty$ almost surely as $t \rightarrow \infty$ for every point $x$, where $d(\cdot, \cdot)$ is the graph distance. This, for example, allows us to define capacities. The general case, which is slightly more complicated to state, is given in Section 7.

Let $V(x, r)$ be the volume of $B(x, r)$ with respect to the measure $\mu(A)=\sum_{x \in A} \mu_{x}$. Let $C(x, r)$ be the capacity of $B(x, r)$ (a definition is given in the next section). Finally define $E(x, r)=V(x, r) / C(x, r)$. It will turn out that $E(x, r)$ is comparable to the expected time that the process spends in $B(x, r)$ when started at $x$.

The novel feature of this paper is to introduce the adjusted Poincaré inequality (API):

$$
\sum_{y \in B(x, r)}\left|f(y)-f_{B(x, r)}\right|^{2} \mu_{y} \leq c E(x, r) \mathcal{E}_{B\left(x, c^{\prime} r\right)}(f, f) .
$$

Here $c^{\prime}>1, f_{A}$ is the average value of $f$ on the set $A$ with respect to the measure $\mu$, and $\mathcal{E}_{A}$ is the Dirichlet form restricted to the set $A$. Note that in the usual Poincaré inequality, $E(x, r)$ is replaced by $r^{\beta}$ for $\beta$ equal to some constant, most often, $\beta=2$.

We will also use another inequality, which we call the cut-off inequality (COI). This is closely related to the cut-off Sobolev inequality of [3].

Our first main theorem is that if transience and regularity hold, then the EHI holds if and only if both the COI and API hold. This immediately implies our second theorem, the stability result, which says that if transience and regularity hold and the EHI holds for a weighted graph, then the EHI holds for every equivalent weighted graph. These results are new even when sufficiently many nice cut-off functions exist.

In the next section we give a precise statement of our results. In Section 3 we introduce the cable process and also prepare some preliminary results. Section 4 proves some estimates that can be obtained from the EHI. We prove that the EHI implies the API in Section 5, and prove our main theorems in Section 6. In Section 7 we consider the general case (where $X$ is not necessarily transient). In that section we also consider extensions to the situation where the state space is a metric measure space rather than a graph.

\section{Statement of results}

We use the letter $c$ with subscripts to denote finite positive constants whose exact values are unimportant and may change from place to place. 
Let $\mathcal{G}$ be an infinite connected graph consisting of vertices $\mathcal{V}$ together with a collection of edges. We write $x \sim y$ if $x$ and $y$ are vertices connected by an edge. We suppose each vertex belongs to at most finitely many edges. For each pair $x, y \in \mathcal{V}$ we define a conductance $C_{x y} \geq 0$ such that $C_{x y}=C_{y x}$ and also $C_{x y}=0$ unless $x \sim y$. The graph $\mathcal{G}$ together with the conductances $\left\{C_{x y}\right\}$ is called a weighted graph.

Let $\mu_{x}=\sum_{y} C_{x y}$, and define a measure $\mu$ on $\mathcal{V}$ by $\mu(A)=\sum_{x \in A} \mu_{x}$. We let $d(x, y)$ be the usual graph distance on $\mathcal{G}$ and set

$$
B(x, r)=\{y: d(x, y)<r\}, \quad V(x, y)=\mu(B(x, r)) .
$$

We assume throughout this paper that there exists a constant $c_{1}$ such that

$$
0<\mu_{x} \leq c_{1}, \quad x \in \mathcal{V} .
$$

For $f \in L^{2}(\mathcal{V}, \mu)$, define

$$
\mathcal{E}_{\mathcal{G}}(f, f)=\frac{1}{2} \sum_{x \sim y}[f(y)-f(x)]^{2} C_{x y}
$$

and

$$
\mathcal{F}_{\mathcal{G}}=\left\{f \in L^{2}(\mathcal{V}, \mu): \mathcal{E}_{\mathcal{G}}(f, f)<\infty\right\} .
$$

It is well known (see [8]) that $\left(\mathcal{E}_{\mathcal{G}}, \mathcal{F}_{\mathcal{G}}\right)$ is a regular Dirichlet form associated with a strong Markov process $\left(X_{t}, \mathbb{P}^{x}\right)$. The process $X$ is a continuous time nearest neighbor Markov chain on $\mathcal{V}$ which can be described as follows. When $X$ is at a vertex $x$, it waits there an independent exponential length of time with parameter $\mu_{x}$ and then jumps to a neighboring vertex. It chooses the neighboring vertex $y$ to jump to with probability $C_{x y} / \mu_{x}$. The infinitesimal generator of $X$ is given by

$$
\mathcal{L}_{\mathcal{G}} f(x)=\sum_{x \sim y}[f(y)-f(x)] C_{x y} .
$$

Except for Section 7 we make a transience assumption.

Assumption 2.1. $\left(\mathcal{E}_{\mathcal{G}}, \mathcal{F}_{\mathcal{G}}\right)$ is transient in the sense of [8, Sect. 1.5].

An equivalent formulation in our context is that

$$
\lim _{t \rightarrow \infty} d\left(X_{t}, x\right)=\infty
$$

with probability one for each starting point and each $x \in \mathcal{V}$.

Let

$$
C(x, r)=\inf \left\{\mathcal{E}_{\mathcal{G}}(f, f): f \in \mathcal{F}_{\mathcal{G}},\left.f\right|_{B(x, r)}=1\right\}
$$

be the capacity of $B(x, r)$. This exists and is finite because $\left(\mathcal{E}_{\mathcal{G}}, \mathcal{F}_{\mathcal{G}}\right)$ is transient; see [8, Sect. 2.1]. Define

$$
E(x, r)=V(x, r) / C(x, r) .
$$

We will see later that $E(x, r)$ is comparable to the expected occupation time of $B(x, r)$ by $X_{t}$ when started at $x$.

Our second main assumption concerns regularity. 
Assumption 2.2. There exist $c_{1}>0$ and $\rho \in(0,1)$ such that the following three inequalities hold:

Volume doubling: $\quad V(x, 2 r) \leq c_{1} V(x, r), \quad x \in \mathcal{V}, r \geq 1$,

Capacity growth: $\quad C(x, r) \leq \rho C(x, 2 r), \quad x \in \mathcal{V}, r \geq 1$,

Expected occupation time growth: $\quad E(x, r) \leq \rho E(x, 2 r), \quad x \in \mathcal{V}, r \geq 1$.

Regarding our assumptions, we make these remarks.

Remark 2.3. See Section 7 for a substitute for Assumption 2.2 when transience is no longer assumed.

Remark 2.4. We will see in the next section that Assumption 2.2 implies $E(x, r)$ and $E(y, r)$ are comparable if $d(x, y) \approx r$, but gives no useful bounds when $d(x, y) \gg r$.

Remark 2.5. We will see in Proposition 4.2 that $E(x, r)$ is comparable to the expected amount of time spent in the ball $B(x, r)$ when the process is started at $x$. The expected occupation time growth condition essentially says that of the time that the process spends in $B(x, 2 r)$, not all of the time is spent in $B(x, r)$ but some percentage of the time is spent in $B(x, 2 r)-B(x, r)$ as well.

Given $f \in \mathcal{F}_{\mathcal{G}}$ and $A \subset \mathcal{V}$, define

$$
\mathcal{E}_{\mathcal{G}, A}=\frac{1}{2} \sum_{x, y \in A}[f(y)-f(x)]^{2} C_{x y},
$$

the Dirichlet form restricted to $A$. Set

$$
f_{A}=\frac{1}{\mu(A)} \sum_{x \in A} f(x) \mu_{x} .
$$

We say the adjusted Poincaré inequality (API) holds for $\mathcal{G}$ if there exist $\kappa_{1}>0$ and $\kappa_{2}>1$ such that

$$
\sum_{y \in B(x, r)}\left[f(y)-f_{B(x, r)}\right]^{2} \mu_{y} \leq \kappa_{1} E(x, r) \mathcal{E}_{\mathcal{G}, B\left(x, \kappa_{2} r\right)}(f, f)
$$

whenever $f \in L^{2}(\mathcal{V}, \mu), x \in \mathcal{V}$, and $r \geq 1$.

Remark 2.6. If $\mathcal{V}=\mathbb{Z}^{d}$ with $\mu$ being counting measure and $d \geq 3$, then $V(x, r) \approx r^{d}$, $C(x, r) \approx r^{d-2}$, and $E(x, r) \approx r^{2}$, where " $\approx$ " means the ratio of the two sides is bounded above and below by positive constants. In this case we get the usual Poincare inequality. For a large class of nested fractals, including the Sierpiński gasket and the Sierpiński carpet (see, e.g., [1] and [2]), $V(x, r) \approx r^{d_{f}}, C(x, r) \approx r^{d_{f}-d_{w}}$, and $E(x, r) \approx r^{d_{w}}$, where $d_{f}$ and $d_{w}$ are the fractal and walk dimensions, resp.

We say the cut-off inequality (COI) holds for $\mathcal{G}$ if there exist $\kappa_{3}, \kappa_{4}$, and $\theta$ such that for each $x_{0} \in \mathcal{V}$ and $R \geq 1$ there exists a function $\varphi=\varphi_{x_{0}, R}$ with the following properties: 
(1) $\varphi(x) \geq 1$ for $x \in B\left(x_{0}, R / 2\right)$ and $\varphi(x)=0$ for $x \notin B\left(x_{0}, R\right)$.

(2) For each $x, y \in \mathcal{V}$,

$$
|\varphi(x)-\varphi(y)| \leq \kappa_{3}(d(x, y) / R)^{\theta} .
$$

(3) If $1 \leq s \leq R$ and $z \in \mathcal{V}$, then

$$
\begin{aligned}
\sum_{x \in B(z, s)} f(x)^{2} & \sum_{y}|\varphi(y)-\varphi(x)|^{2} C_{x y} \\
& \leq \kappa_{4}(s / R)^{2 \theta}\left(\mathcal{E}_{\mathcal{G}, B(z, 2 s)}(f, f)+E(z, s)^{-1} \sum_{x \in B(z, 2 s)} f(x)^{2} \mu_{x}\right)
\end{aligned}
$$

for all $f \in \mathcal{F}_{\mathcal{G}}$.

Remark 2.7. The COI is very similar to the CS inequality of [3], where an extensive discussion can be found. See [4] for examples of how the COI can be used to prove Harnack inequalities for graphs that are in rough isometry to simpler graphs. Further research on finding conditions equivalent to COI would be highly desirable.

We say a function $h$ on a subset $D$ of $\mathcal{V}$ is harmonic if

$$
\mathcal{L} h(x)=0, \quad x \in D .
$$

This is equivalent to

$$
h(x)=\sum_{y} h(y) C_{x y}, \quad x \in D .
$$

The elliptic Harnack inequality (EHI) holds for the weighted graph $\mathcal{G}$ with conductances $\left\{C_{x y}\right\}$ if there exists $c_{1}$ such that whenever $x_{0} \in \mathcal{V}, r \geq 1$, and $h$ is non-negative and harmonic in $B\left(x_{0}, 2 r\right)$, then

$$
h(x) \leq c_{1} h(y), \quad x, y \in B\left(x_{0}, r\right) .
$$

Our first main theorem is the following.

Theorem 2.8. Suppose (2.1) and Assumptions 2.1 and 2.2 hold.

(a) If the EHI holds for $\mathcal{G}$, then both the API and COI hold for $\mathcal{G}$.

(b) If the API and COI hold for $\mathcal{G}$, then the EHI holds for $\mathcal{G}$.

Suppose we have another set of conductances $\left\{C_{x y}^{\prime}\right\}$ on the graph $\mathcal{G}$. We say $\left(\mathcal{G}, C_{x y}\right)$ and $\left(\mathcal{G}, C_{x y}^{\prime}\right)$ are equivalent weighted graphs if there exists $c_{1}<1$ such that

$$
c_{1} C_{x y} \leq C_{x y}^{\prime} \leq c_{1}^{-1} C_{x y}, \quad x, y \in \mathcal{V} .
$$

Our second main theorem is the stability theorem.

Theorem 2.9. Suppose $\left(\mathcal{G}, C_{x y}\right)$ and $\left(\mathcal{G}, C_{x y}^{\prime}\right)$ are equivalent weighted graphs. Suppose (2.1) and Assumptions 2.1 and 2.2 hold for $\left(\mathcal{G}, C_{x y}\right)$ and for $\left(\mathcal{G}, C_{x y}^{\prime}\right)$. If the EHI holds for $\left(\mathcal{G}, C_{x y}\right)$, then the EHI holds for $\left(\mathcal{G}, C_{x y}^{\prime}\right)$.

See Section 7 for a statement of these theorems in the context of metric measure spaces or when Assumption 2.1 does not hold. 


\section{Preliminaries}

We introduce the cable process. Let $\mathcal{C}$ consist of $\mathcal{V}$ together with copies of $(0,1)$, one for each edge in $\mathcal{G}$. If $x \sim y$, we write $(x, y)$ for the corresponding copy, and we call $(x, y)$ the cable connecting $x$ and $y$. We identify $x$ with 0 and $y$ with 1 on the cable connecting $x$ and $y$. We define $\mu(d z)$ by setting it equal to $C_{x y} d z$ on the cable connecting $x$ and $y$, where $d z$ is linear Lebesgue measure. If $x$ and $y$ are two points on the same cable or one lies on a cable and the other is an endpoint of that cable, then we define the distance between $x$ and $y$ to be $|x-y|$. If $x$ and $y$ are on different cables, we use $\min \left\{\left|x-z_{x}\right|+d\left(z_{x}, z_{y}\right)+\left|z_{y}-y\right|\right\}$ for the distance, where the minimum is taken over all vertices $z_{x}, z_{y} \in \mathcal{V}$ such that $x$ is on a cable with one end at $z_{x}$ and $y$ is on a cable with one end at $z_{y}$. We continue to use the notation $d(x, y)$ for the distance and set

$$
B^{\prime}(x, r)=\{y \in \mathcal{C}: d(x, y)<r\}, \quad V^{\prime}(x, r)=\mu\left(B^{\prime}(x, r)\right) .
$$

The cable process is the process that behaves like one-dimensional Brownian motion sped up deterministically by the factor $C_{x y}$ on $(x, y)$ and when at a vertex $x$, picks the cable along which the next excursion takes place according to the probabilities $C_{x y} / \mu_{x}$. More precisely, if $x \in \mathcal{C}-\mathcal{V}$ and $x$ lies on the cable $\left(y_{0}, y_{1}\right)$, let

$$
\nabla f(x)=\lim _{z \rightarrow x} \frac{f(z)-f(x)}{d\left(y_{0}, z\right)-d\left(y_{0}, x\right)} .
$$

If $x \in \mathcal{V}$ and $x \sim y$, let

$$
\nabla_{y} f(x)=\lim _{z \rightarrow x, z \in(x, y)} \frac{f(z)-f(x)}{d(x, z)} .
$$

Since we only work with $|\nabla f|$ and $\left|\nabla_{y} f\right|$, we do not need to be concerned with whether we use $y_{0}$ or $y_{1}$ in the definition of $\nabla f(x)$. Let

$$
\mathcal{E}_{\mathcal{C}}(f, f)=\frac{1}{2} \int_{\mathcal{C}-\mathcal{V}}|\nabla f(z)|^{2} \mu(d z),
$$

let $\mathcal{F}_{\mathcal{C}}^{0}$ be the collection of continuous functions with compact support such that $\nabla f(z)$ exists at every point of $\mathcal{C}-\mathcal{V}, \nabla_{y} f(x)$ exists at every $x \in \mathcal{V}$ for which $y \sim x$, and $|\nabla f|$ is bounded. For the domain of $\mathcal{E}_{\mathcal{C}}$, we use $\mathcal{F}_{\mathcal{C}}$, which is the completion of $\mathcal{F}_{\mathcal{C}}^{0}$ with respect to the norm

$$
\left(\int_{\mathcal{C}}|f(z)|^{2} \mu(d z)\right)^{1 / 2}+\mathcal{E}_{\mathcal{C}}(f, f)^{1 / 2} .
$$

The cable process is the symmetric continuous Markov process $\left(Y_{t}, \mathbb{P}^{x}\right)$ corresponding to $\left(\mathcal{E}_{\mathcal{C}}, \mathcal{F}_{\mathcal{C}}\right)$. Typically when constructing a process via Dirichlet forms, there is a null set involved, and one has to talk about properties holding quasi-everywhere. However, in our case $\mathbb{P}^{x}\left(Y_{t}\right.$ ever hits $\left.y\right)>0$ for each $x$ and $y$, and no null set is necessary.

Let $\mathcal{L}_{\mathcal{C}}$ be the infinitesimal generator of $Y$. See [3] for a detailed description of $\mathcal{L}_{\mathcal{C}}$ and its domain. 
Proposition 3.1. Suppose (2.1) and Assumptions 2.1 and 2.2 hold. Then $\left(\mathcal{E}_{\mathcal{C}}, \mathcal{F}_{\mathcal{C}}\right)$ is transient. Let

$$
C^{\prime}(x, r)=\inf \left\{\mathcal{E}_{\mathcal{C}}(f, f):\left.f\right|_{B^{\prime}(x, r)}=1, f \in \mathcal{F}_{\mathcal{C}}\right\}
$$

be the capacity of $B^{\prime}(x, r)$ and let

$$
E^{\prime}(x, r)=\frac{V^{\prime}(x, r)}{C^{\prime}(x, r)} .
$$

Then there exist $c_{1}>0$ and $\rho \in(0,1)$ such that

$$
\begin{aligned}
V^{\prime}(x, 2 r) & \leq c_{1} V^{\prime}(x, r), \\
C^{\prime}(x, r) & \leq \rho C^{\prime}(x, 2 r), \\
E^{\prime}(x, r) & \leq \rho E^{\prime}(x, 2 r)
\end{aligned}
$$

whenever $x \in \mathcal{C}$ and $r>0$. Moreover there exists a positive integer $M$ not depending on $x$ or $r$ such that the boundary of $B^{\prime}(x, r)$ can be covered by at most $M$ balls of radius $r / 8$.

Proof. The assertions (3.1), (3.2), and (3.3) follow easily by using the techniques of [3, Section 3] and we leave the details to the reader. For the assertion about $M$, see [11, Lemma 3.1].

Given $f \in \mathcal{F}_{\mathcal{C}}$ and $A \subset \mathcal{C}$, define

$$
\mathcal{E}_{\mathcal{C}, A}=\frac{1}{2} \int_{A-\mathcal{V}}|\nabla f(x)|^{2} \mu(d x) .
$$

and set

$$
f_{A}=\frac{1}{\mu(A)} \int_{A} f(x) \mu(d x) .
$$

We say the adjusted Poincaré inequality (API) holds for $\mathcal{C}$ if there exist $\kappa_{1}>0$ and $\kappa_{2}>1$ such that

$$
\int_{B^{\prime}(x, r)}\left[f(y)-f_{B^{\prime}(x, r)}\right]^{2} \mu(d y) \leq \kappa_{1} E^{\prime}(x, r) \mathcal{E}_{\mathcal{C}, B^{\prime}\left(x, \kappa_{2} r\right)}(f, f)
$$

whenever $f \in \mathcal{F}_{\mathcal{C}}, x \in \mathcal{C}$.

We say the cut-off inequality (COI) holds for $\mathcal{C}$ if there exist $\kappa_{3}, \kappa_{4}$, and $\theta$ such that for each $x_{0} \in \mathcal{C}$ and $R>0$ there exists a function $\varphi=\varphi_{x_{0}, R}$ with the following properties.

(1) $\varphi(x) \geq 1$ for $x \in B^{\prime}\left(x_{0}, R / 2\right)$ and $\varphi(x)=0$ for $x \notin B^{\prime}\left(x_{0}, R\right)$.

(2) For each $x, y \in \mathcal{C}$,

$$
|\varphi(x)-\varphi(y)| \leq \kappa_{3}(d(x, y) / R)^{\theta} .
$$


(3) If $0 \leq s \leq R$ and $z \in \mathcal{C}$, then

$$
\begin{aligned}
\int_{B^{\prime}(z, s)} & f(x)^{2}|\nabla \varphi(x)|^{2} \mu(d x) \\
& \leq \kappa_{4}(s / R)^{2 \theta}\left(\mathcal{E}_{\mathcal{C}, B^{\prime}(z, 2 s)}(f, f)+E^{\prime}(z, s)^{-1} \int_{B^{\prime}(z, 2 s)} f(x)^{2} \mu(d x)\right)
\end{aligned}
$$

for all $f \in \mathcal{F}_{\mathcal{C}}$.

We say a function $h$ in the domain of $\mathcal{L}_{\mathcal{C}}$ is harmonic on a subset $D$ of $\mathcal{C}$ if

$$
\mathcal{L}_{\mathcal{C}} h(x)=0, \quad x \in D .
$$

The elliptic Harnack inequality (EHI) holds for $\mathcal{C}$ if there exists $c_{1}$ such that whenever $x_{0} \in \mathcal{C}, r>0$, and $h$ is non-negative and harmonic in $B^{\prime}\left(x_{0}, 2 r\right)$, then

$$
h(x) \leq c_{1} h(y), \quad x, y \in B^{\prime}\left(x_{0}, r\right) .
$$

Proposition 3.2. (a) The COI holds for $\mathcal{C}$ if and only the COI holds for $\mathcal{G}$.

(b) The API holds for $\mathcal{C}$ if and only the API holds for $\mathcal{G}$.

(c) The EHI holds for $\mathcal{C}$ if and only the EHI holds for $\mathcal{G}$.

Proof. The proof of (a) is almost identical to that of Propositions 3.3 and 3.4 of [3]. The same techniques can be used to prove (b). Finally, (c) is [3, Cor. 2.5].

The main work in this paper is to prove the following.

Theorem 3.3. Suppose (2.1) and Assumptions 2.1 and 2.2 hold.

(a) If the EHI holds for $\mathcal{C}$, then both the API and COI hold for $\mathcal{C}$.

(b) If both the API and COI hold for $\mathcal{C}$, then the EHI holds for $\mathcal{C}$.

It will be clear from the context whether we are working with $\mathcal{C}$ or $\mathcal{G}$, so henceforth we will drop the primes and write $B(x, r), V(x, r), C(x, r)$, and $E(x, r)$ in place of $B^{\prime}(x, r)$, $V^{\prime}(x, r), C^{\prime}(x, r)$, and $E^{\prime}(x, r)$, resp. We write $\partial B(x, r)$ for the boundary of $B(x, r)$.

Lemma 3.4. There exist $c_{1}>0$ and $\rho^{\prime} \in(0,1)$ such that volume growth holds:

$$
V(x, r) \leq \rho^{\prime} V(x, 2 r), \quad x \in \mathcal{C}, r>0 ;
$$

capacity doubling holds:

$$
C(x, 2 r) \leq c_{1} C(x, r), \quad x \in \mathcal{C}, r>0 ;
$$

and expected occupation time doubling holds:

$$
E(x, 2 r) \leq c_{1} E(x, r), \quad x \in \mathcal{C}, r>0 .
$$


Proof. Multiplying (3.2) and (3.3) together gives volume growth. Expected occupation time growth implies

$$
C(x, 2 r) \leq \rho C(x, r) \frac{V(x, 2 r)}{V(x, r)},
$$

and an application of volume doubling implies capacity doubling. Finally, since $C(x, r) \leq$ $C(x, 2 r)$, volume doubling implies

$$
\frac{V(x, 2 r)}{C(x, 2 r)} \leq c_{2} \frac{V(x, r)}{C(x, r)},
$$

which is expected occupation time doubling.

Lemma 3.5. Let $a>0$. There exists $c_{1}$ depending on a but not on $r, x$, or y such that if $d(x, y)<$ ar, then

$$
V(x, r) \leq c_{1} V(y, r), \quad C(x, r) \leq c_{1} C(y, r), \quad E(x, r) \leq c_{1} E(y, r) .
$$

Proof. Since $B(x, r) \subset B(y,(1+a) r)$, volume doubling tells us

$$
V(x, r) \leq V(y,(1+a) r) \leq c_{2} V(y, r),
$$

and similarly for $V$ replaced by $C$. By symmetry, $C(y, r) \leq c_{2} C(x, r)$, so taking the ratio, $E(x, r) \leq c_{2}^{2} E(y, r)$.

In Proposition 3.1 we may without loss of generality assume that the center of each of the $M$ balls is within $r / 8$ of $\partial B(x, r)$. If we let $B_{1}, \ldots, B_{M}$ be balls with the same centers but radii equal to $r / 4$, then for each $j \geq 2$, there exists $i<j$ and a point $y_{j}$ such that $y_{j} \in B_{i} \cap B_{j}$. If $h$ is non-negative and harmonic in $B(x, 2 r)-B(x, r / 2)$ and the EHI holds, then for $w \in B_{i}$ and $z \in B_{j}$,

$$
h(w) \leq c_{1} h\left(y_{j}\right) \leq c_{1}^{2} h(z) .
$$

Using this inequality at most $M$ times, where $M$ is given in Proposition 3.1, we find that there is a constant $c_{2}$ such that if $y, z \in \partial B(x, r)$, then

$$
h(y) \leq c_{2} h(z) .
$$

Let $G(x, y)$ be the Green function for the process $Y_{t}$. The existence of $G$ is an easy consequence of Assumption 2.1 and the structure of $\mathcal{C}$. For $x$ fixed, $h(z)=G(x, z)$ is a non-negative function that is harmonic in $B(x, 2 r)-B(x, r / 2)$ and so we may apply (3.10) to $G(x, \cdot)$ and obtain

$$
G(x, y) \leq c_{2} G(x, z), \quad y, z \in \partial B\left(x_{0}, r\right) .
$$

When the EHI holds, harmonic functions are Hölder continuous (see [12]), and so there exist $c_{3}$ and $\beta$ such that if $h$ is harmonic in $B\left(x_{0}, 2 r\right)$, then

$$
|h(x)-h(y)| \leq c_{3}\left(\frac{d(x, y)}{r}\right)^{\beta}\left(\sup _{B\left(x_{0}, 2 r\right)}|h|\right), \quad x, y \in B\left(x_{0}, r\right) .
$$




\section{Some consequences of the EHI}

In this section we assume the EHI holds for a process $Y$ associated with a Dirichlet form $(\mathcal{E}, \mathcal{F})$.

The first estimate is standard. Let $G(x, y)$ be the Green function for $Y$.

Proposition 4.1. There exist constants $c_{1}$ and $c_{2}$ such that if $r=d(x, y)$, then

$$
c_{1} / C(x, r) \leq G(x, y) \leq c_{2} / C(x, r), \quad x \in \mathcal{C}, r>0 .
$$

Proof. Let $x$ and $y$ be fixed and let $r=d(x, y)$. Let $v$ be the capacitary measure for $B(x, r)$. Then we know $v$ is supported on $\partial B(x, r)$, its total mass is $C(x, r)$, and $G v$ equals 1 on $B(x, r)$. (See [5, Section II.5], for example. The proofs there are for Brownian motion but are valid for any symmetric continuous strong Markov process.) Using (3.11), we may write

$$
1=G v(x)=\int_{\partial B(x, r)} G(x, z) v(d z) \geq c_{3} G(x, y) \int_{\partial B(x, r)} v(d z)=c_{3} G(x, y) C(x, r) .
$$

Rearranging gives the right hand inequality. The left hand inequality is proved in the same way, replacing " $\geq$ " by " $\leq$."

Next we obtain an estimate on the time spent in $B(x, r)$.

Proposition 4.2. There exist constants $c_{1}$ and $c_{2}$ such that

$$
c_{1} E(x, r) \leq \int_{B(x, r)} G(x, z) \mu(d z) \leq c_{2} E(x, r) .
$$

Proof. Let $\rho^{\prime}$ be the constant in Lemma 3.4. Applying (3.11), Proposition 4.1, and (3.7), we obtain

$$
\begin{aligned}
\int_{B(x, r)} G(x, z) \mu(d z) & \geq \int_{B(x, r)-B(x, r / 2)} G(x, z) \mu(d z) \\
\geq & \frac{c_{3}}{C(x, r)}(V(x, r)-V(x, r / 2)) \geq \frac{c_{3}\left(1-\rho^{\prime}\right)}{C(x, r)} V(x, r)=c_{4} E(x, r) .
\end{aligned}
$$

This gives the left hand inequality.

Similarly, we have

$$
\int_{B(x, r)-B(x, r / 2)} G(x, z) \mu(d z) \leq c_{5} \frac{V(x, r)-V(x, r / 2)}{C(x, r)} \leq c_{5} E(x, r)
$$

for each $r>0$. We apply this with $r$ replaced by $2^{-k} r$ for $k=0,1, \ldots$, and sum. Using the fact that $Y$ spends 0 time at $x$ (locally $Y$ behaves like a deterministic time change of Brownian motion), we obtain

$$
\int_{B(x, r)} G(x, z) \mu(d z) \leq c_{5} \sum_{k=0}^{\infty} E\left(x, 2^{-k} r\right) .
$$


Using (3.3) repeatedly, we have $E\left(x, 2^{-k} r\right) \leq \rho^{k} E(x, r)$, so

$$
\int_{B(x, r)} G(x, z) \mu(d z) \leq c_{5} E(x, r) \sum_{k=0}^{\infty} \rho^{k},
$$

which implies the right hand inequality.

\section{The adjusted Poincaré inequality}

Let $G_{D}$ denote the Green function for $Y$ killed on exiting a domain $D$.

Proposition 5.1. Suppose (2.1), Assumptions 2.1 and 2.2, and the EHI hold. There exist $k_{0} \geq 2$ and $c_{1}$ not depending on $x_{0}$ or $r$ such that if $r>0$ and $x, y \in B\left(x_{0}, r\right)$, then

$$
G_{B\left(x_{0}, 2^{k} r\right)}(x, y) \geq c_{1} / C\left(x_{0}, r\right) .
$$

Proof. Let $s=d(x, y)$ and note $B(x, s) \subset B\left(x_{0}, 4 r\right)$. By Proposition 4.1 and (3.8), there exists a constant $c_{2}$ such that

$$
G(x, y) \geq c_{2} / C(x, s) \geq c_{2} / C\left(x_{0}, 4 r\right) \geq c_{3} / C\left(x_{0}, r\right) .
$$

By the strong Markov property,

$$
G_{D}(x, y)=G(x, y)-\mathbb{E}^{x} G\left(Y_{\tau_{D}}, y\right),
$$

where $\tau_{D}$ is the first time that $Y$ exits $D$. By (3.11), if $D=B\left(x_{0}, 2^{k} r\right)$ for some $k \geq 1$ and $w \in \partial D$, then

$$
G(w, y) \leq c_{4} G\left(w, x_{0}\right) \leq c_{5} / C\left(x_{0}, 2^{k} r\right) \leq c_{5} \rho^{k} / C\left(x_{0}, r\right),
$$

where $\rho$ is the constant in Proposition 3.1. If we choose $k_{0} \geq 2$ large enough so that $c_{5} \rho^{k_{0}} \leq c_{3} / 2$ and combine (5.1)-(5.3), we obtain our proposition with $c_{1}=c_{3} / 2$.

We write $\left(G_{D}\right)^{2} f$ for $G_{D}\left(G_{D} f\right)$.

Proposition 5.2. Suppose (2.1), Assumptions 2.1 and 2.2, and the EHI hold. Let $k_{0}$ be defined as in Proposition 5.1 and let $D=B\left(x_{0}, 2^{k_{0}} r\right)$. There exists $c_{1}$ not depending on $x_{0}$ or $r$ such that

$$
\left(G_{D}\right)^{2}(x, y) \leq c_{1} E\left(x_{0}, r\right) G_{D}(x, y) \quad \text { for all } x, y \in B\left(x_{0}, r\right) .
$$

Proof. Write

$$
\left(G_{D}\right)^{2}(x, y)=\int G_{D}(x, z) G_{D}(z, y) \mu(d z) .
$$

We let $s=d(x, y)$ (so that $s<2 r$ ) and break the integral on the right into integrals over $B(x, s / 2)$ and over $B(x, s / 2)^{c}$.

For $z \in B(x, s / 2)$, we have $d(z, y) \geq s / 2$, and by (3.11),

$$
G_{D}(z, y) \leq c_{2} G_{D}(x, y) .
$$


Since $D \subset B\left(x, 2^{k_{0}+1} r\right)$, using Proposition 4.2, (3.9), and Lemma 3.5 yields

$$
\begin{aligned}
& \int_{B(x, s / 2)} G_{D}(x, z) G_{D}(z, y) \mu(d z) \leq c_{2} G_{D}(x, y) \int_{D} G_{D}(x, z) \mu(d z) \\
& \quad \leq c_{2} G_{D}(x, y) \int_{D} G(x, z) \mu(d z) \leq c_{3} G_{D}(x, y) \int_{B\left(x, 2^{k_{0}+1} r\right)} G(x, z) \mu(d z) \\
& \quad \leq c_{4} G_{D}(x, y) E\left(x, 2^{k_{0}+1} r\right) \leq c_{5} G_{D}(x, y) E(x, r) \leq c_{6} G_{D}(x, y) E\left(x_{0}, r\right) .
\end{aligned}
$$

For $z \in B(x, s / 2)^{c}$, we have $d(z, x) \geq s / 2$, and by (3.11),

$$
G_{D}(x, z) \leq c_{2} G_{D}(x, y) .
$$

As above, using that $G_{D}$ is zero on $D^{c}$, we obtain

$$
\begin{aligned}
\int_{B(x, s / 2)^{c}} G_{D} & (x, z) G_{D}(z, y) \mu(d z) \leq c_{2} G_{D}(x, y) \int_{D} G_{D}(y, z) \mu(d z) \\
& \leq c_{2} G_{D}(x, y) \int_{D} G(y, z) \mu(d z) \leq c_{2} G_{D}(x, y) \int_{B\left(y, 2^{k_{0}+1} r\right)} G(y, z) \mu(d z) \\
& \leq c_{7} G_{D}(x, y) E\left(y, 2^{k_{0}+1} r\right) \leq c_{8} G_{D}(x, y) E(y, r) \leq c_{9} G_{D}(x, y) E\left(x_{0}, r\right) .
\end{aligned}
$$

In the third inequality we used the fact that $D \subset B\left(y, 2^{k_{0}+1} r\right)$, and we used Lemma 3.5 for the last inequality. Adding the integrals over $B(x, s / 2)$ and $B(x, s / 2)^{c}$ yields our result.

Let $G^{\alpha}$ be the $\alpha$-resolvent for $Y$, and $G_{D}^{\alpha}$ the $\alpha$-resolvent for the process killed on exiting $D$.

Proposition 5.3. Suppose (2.1), Assumptions 2.1 and 2.2, and the EHI hold. Let D be as in Proposition 5.2. There exist $c_{1}, c_{2}$ not depending on $x_{0}$ or $r$ such that if $\alpha=$ $c_{1} / E\left(x_{0}, r\right)$ and $x, y \in B\left(x_{0}, r\right)$, then

$$
G_{D}^{\alpha}(x, y) \geq c_{2} / C\left(x_{0}, r\right) .
$$

Proof. By the resolvent equation, $G_{D}^{\alpha}=G_{D}-\alpha G_{D} G_{D}^{\alpha}$, and so

$$
G_{D}^{\alpha}(x, y)=G_{D}(x, y)-\alpha G_{D} G_{D}^{\alpha}(x, y) \geq G_{D}(x, y)-\alpha\left(G_{D}\right)^{2}(x, y) .
$$

From Proposition 5.2 we know

$$
\left(G_{D}\right)^{2}(x, y) \leq c_{3} E\left(x_{0}, r\right) G_{D}(x, y)
$$

for $x, y \in B\left(x_{0}, r\right)$. By Proposition 5.1 we also know $G_{D}(x, y) \geq c_{4} / C\left(x_{0}, r\right)$. Then

$$
G_{D}^{\alpha}(x, y) \geq G_{D}(x, y)\left(1-\alpha c_{3} E\left(x_{0}, r\right)\right) \geq \frac{c_{4}}{C\left(x_{0}, r\right)}\left(1-\alpha c_{3} E\left(x_{0}, r\right)\right) .
$$

If we take $c_{1}=\left(2 c_{3}\right)^{-1}$, then since $\alpha=c_{1} / E\left(x_{0}, r\right)$, we have $1-\alpha c_{3} E\left(x_{0}, r\right) \geq 1 / 2$, and our result follows. 
Given a ball $D$, we let $Y^{r}$ be the process $Y$ reflected on the boundary of $D$. Since $Y$ behaves locally like a Brownian motion, it is clear how $Y^{r}$ can be described probabilistically. Using a more analytic approach, $Y^{r}$ is the continuous symmetric strong Markov process corresponding to $\mathcal{E}_{D}$ with domain $\left\{f \in \mathcal{F}: \int_{D}\left(|f|^{2}+|\nabla f|^{2}\right)<\infty\right\}$.

Theorem 5.4. Suppose (2.1) and Assumptions 2.1 and 2.2 hold. If the EHI holds for $\mathcal{C}$, then the API holds for $\mathcal{C}$.

Proof. Fix $x_{0}$ and $r>0$. Let $B=B\left(x_{0}, r\right)$ and $D=B\left(x_{0}, 2^{k_{0}} r\right)$, where $k_{0}$ is as in Proposition 5.1. Let $\alpha$ be as in Proposition 5.3. Let $Y^{r}$ be the process $Y$ reflected on the boundary of $D$, and let $G_{r}^{\alpha}$ be the $\alpha$-resolvent for $Y^{r}$. Fix $f \in L^{2}(D) \cap \mathcal{F}$. Take $x \in B$. Since the quantity $\int_{B}(f(y)-c)^{2} \mu(d y)$ takes its minimum when $c=f_{B}$, it follows that

$$
\int_{B}\left(f(y)-f_{B}\right)^{2} \mu(d y) \leq \int_{B}\left(f(y)-\alpha G_{r}^{\alpha} f(x)\right)^{2} \mu(d y) .
$$

For $x, y \in B$, we have

$$
\alpha G_{r}^{\alpha}(x, y) \geq \alpha G_{D}^{\alpha}(x, y) \geq \frac{c_{1}}{E\left(x_{0}, r\right) C\left(x_{0}, r\right)} \geq \frac{c_{1}}{V\left(x_{0}, r\right)} .
$$

For any function $h$,

$$
\alpha G_{r}^{\alpha} h(x)=\int_{D} h(y) \alpha G_{r}^{\alpha}(x, y) \mu(d y) \geq \frac{c_{1}}{V\left(x_{0}, r\right)} \int_{B} h(y) \mu(d y) .
$$

Using (5.4) and letting $h(y)=\left(f(y)-\alpha G_{r}^{\alpha} f(x)\right)^{2}$, we obtain

$$
\begin{aligned}
\int_{B}\left(f(y)-f_{B}\right)^{2} \mu(d y) & \leq c_{2} V\left(x_{0}, r\right)\left[\alpha G_{r}^{\alpha}\left(\left(f(\cdot)-\alpha G_{r}^{\alpha} f(x)\right)^{2}\right)(x)\right] \\
& =c_{2} V\left(x_{0}, r\right)\left[\alpha G_{r}^{\alpha}\left(f^{2}\right)(x)-\left(\alpha G_{r}^{\alpha} f(x)\right)^{2}\right] .
\end{aligned}
$$

The right hand side is non-negative. Integrating both sides over the set $D$ with respect to the measure $\mu(d x)$, multiplying by $\mu(B)^{-1}$, and using volume doubling gives

$$
\int_{B}\left(f(y)-f_{B}\right)^{2} \mu(d y) \leq c_{2}\left[\int_{D} \alpha G_{r}^{\alpha}(f)^{2}(x) \mu(d x)-\int_{D}\left(\alpha G_{r}^{\alpha} f(x)\right)^{2} \mu(d x)\right] .
$$

If $\langle\cdot, \cdot\rangle$ is the inner product with respect to $L^{2}(D)$, then using the symmetry of the resolvent, the first integral inside the brackets on the last line is

$$
\left\langle\alpha G_{r}^{\alpha}\left(f^{2}\right), 1\right\rangle=\left\langle f^{2}, \alpha G_{r}^{\alpha} 1\right\rangle=\left\langle f^{2}, 1\right\rangle=\|f\|_{2}^{2},
$$

where we write $\|\cdot\|_{2}$ for the $L^{2}$ norm on $D$. The second integral on the right hand side of (5.6) is $\left\|\alpha G_{r}^{\alpha} f\right\|_{2}^{2}$, and we thus have

$$
\int_{B}\left(f(y)-f_{B}\right)^{2} \mu(d y) \leq c_{2}\left[\|f\|_{2}^{2}-\left\|\alpha G_{r}^{\alpha} f\right\|_{2}^{2}\right] .
$$


We now use the spectral theorem for $L^{2}(D)$. Let $\left\{E_{\lambda}\right\}$ be the spectral resolution of the operator $\mathcal{L}^{r}$, the infinitesimal generator of $Y^{r}$. Each $E_{\lambda}$ is a projection, and we can write

$$
f=\int_{0}^{\infty} d E_{\lambda} f, \quad\|f\|_{2}^{2}=\int_{0}^{\infty} d\left\langle E_{\lambda} f, E_{\lambda} f\right\rangle .
$$

For $f \in \mathcal{F}$, we have

$$
\mathcal{E}_{D}(f, f)=\int_{0}^{\infty} \lambda d\left\langle E_{\lambda} f, E_{\lambda} f\right\rangle
$$

We also have

$$
\alpha G_{r}^{\alpha} f=\int_{0}^{\infty} \frac{\alpha}{\alpha+\lambda} d E_{\lambda} f, \quad\left\|\alpha G_{r}^{\alpha} f\right\|_{2}^{2}=\int_{0}^{\infty}\left(\frac{\alpha}{\alpha+\lambda}\right)^{2} d\left\langle E_{\lambda} f, E_{\lambda} f\right\rangle .
$$

Since

$$
1-\left(\frac{\alpha}{\alpha+\lambda}\right)^{2}=\frac{2 \lambda(\alpha+\lambda / 2)}{(\alpha+\lambda)^{2}} \leq \frac{2 \lambda}{\alpha}
$$

we have

$$
\begin{aligned}
\|f\|_{2}^{2}-\left\|\alpha G_{r}^{\alpha} f\right\|_{2}^{2} & =\int_{0}^{\infty}\left(1-\left(\frac{\alpha}{\alpha+\lambda}\right)^{2}\right) d\left\langle E_{\lambda} f, E_{\lambda} f\right\rangle \\
& \leq c_{2} \frac{2}{\alpha} \int_{0}^{\infty} \lambda d\left\langle E_{\lambda} f, E_{\lambda} f\right\rangle=c_{3} E\left(x_{0}, r\right) \mathcal{E}_{D}(f, f) .
\end{aligned}
$$

Combining (5.7) and (5.8) proves the API.

\section{Proofs of main theorems}

Throughout we assume (2.1) and Assumptions 2.1 and 2.2. We continue the cable system context unless stated otherwise.

We need two propositions which will be used to show that the COI and API imply the EHI.

Fix $x_{0} \in \mathcal{C}$, let $R \geq 1$, and let $\varphi$ be the cut-off function given by the COI. Let

$$
\gamma=1+E\left(x_{0}, R\right)|\nabla \varphi|^{2} .
$$

Proposition 6.1. Suppose the API holds for $\mathcal{C}$ with constants $\kappa_{1}$ and $\kappa_{2}$ and also the COI holds. Let $x \in B\left(x_{0}, R\right)$, let $I=B(x, s)$ with $s \leq R$, and let $I^{*}=B(x, 2 s)$, $I^{* *}=B\left(x, 2 \kappa_{2} s\right)$. Suppose $f$ and its gradient are square integrable over $I^{* *}$ and let $f_{A}=\mu(A)^{-1} \int_{A} f d \mu$. Then

$$
\int_{I} f^{2} \gamma \leq c_{1}(s / R)^{2 \theta} E\left(x_{0}, R\right)\left(\int_{I^{*}}|\nabla f|^{2}+E(x, s)^{-1} \int_{I^{*}} f^{2}\right)
$$

and

$$
\int_{I}\left(f-f_{I^{*}}\right)^{2} \gamma \leq c_{2}(s / R)^{2 \theta} E\left(x_{0}, R\right) \int_{I^{* *}}|\nabla f|^{2} .
$$


If $J \subset I$, then

$$
\int_{J} f^{2} \gamma \leq c_{3}\left(E\left(x_{0}, R\right)(s / R)^{2 \theta}\right) \int_{I^{* *}}|\nabla f|^{2}+\mu(J)^{-1}\left(\int_{J}|f| \gamma\right)^{2} .
$$

Finally,

$$
\int_{B\left(x_{0}, R\right)} \gamma \leq c_{4} V\left(x_{0}, R\right) .
$$

Proof. The condition (3.3) implies that $E(x, R) / E(x, s) \geq c_{5}(R / s)^{\beta}$ for some $\beta>0$ and $c_{5}>0$ not depending on $x, R$, or $s$. Without loss of generality we may assume $2 \theta<\beta$. Then

$$
(s / R)^{2 \theta} E(x, R) E(x, s)^{-1} \geq c_{6}
$$

since $s \leq R$. Using Lemma 3.5, $E\left(x_{0}, R\right) \geq c_{7} E(x, R)$ and hence

$$
\begin{aligned}
\int_{I} f^{2} \gamma & =\int_{I} f^{2}+E\left(x_{0}, R\right) \int_{I} f^{2}|\nabla \varphi|^{2} \\
& \leq \int_{I} f^{2}+c_{8}(s / R)^{2 \theta} E\left(x_{0}, R\right) \int_{I^{*}}|\nabla f|^{2}+c_{8}(s / R)^{2 \theta} \frac{E\left(x_{0}, R\right)}{E(x, s)} \int_{I^{*}} f^{2} \\
& \leq c_{9}(s / R)^{2 \theta} E\left(x_{0}, R\right) \int_{I^{*}}|\nabla f|^{2}+c_{9}(s / R)^{2 \theta} \frac{E\left(x_{0}, R\right)}{E(x, s)} \int_{I^{*}} f^{2} .
\end{aligned}
$$

Applying this to $f-f_{I^{*}}$, we have

$$
\int_{I}\left(f-f_{I^{*}}\right)^{2} \gamma \leq c_{10}(s / R)^{2 \theta} E\left(x_{0}, R\right)\left(\int_{I^{*}}|\nabla f|^{2}+E(x, s)^{-1} \int_{I^{*}}\left(f-f_{I^{*}}\right)^{2}\right) .
$$

Applying the API to $B(x, 2 s)$ yields

$$
E(x, s)^{-1} \int_{I^{*}}\left(f-f_{I^{*}}\right)^{2} \leq c_{11} \int_{I^{* *}}|\nabla f|^{2} .
$$

Combining gives (6.2).

The remainder of the proof is exactly as in [3, Prop. 5.2].

Here is a substitute for [3, Prop. 5.7].

Proposition 6.2. Suppose the API holds for $\mathcal{C}$ with constants $\kappa_{1}$ and $\kappa_{2}$ and also the COI holds. Let $S>0$, let $u$ be positive and harmonic in $B\left(x_{0}, 2 \kappa_{2} S\right)$ and let $w=\log u$. Then

$$
\int_{B\left(x_{0}, 2 S\right)}|\nabla w|^{2} d \mu \leq c_{1} C\left(x_{0}, S\right) .
$$


Proof. Let $\varphi_{1}$ be the cut-off function for $B\left(x_{0}, 2 \kappa_{2} S\right)$ given by the COI. Exactly as in the proof of [3, Prop. 5.7] we have

$$
\int_{B\left(x_{0}, 2 S\right)}|\nabla w|^{2} d \mu \leq \int \varphi_{1}^{2}|\nabla w|^{2} d \mu \leq c_{2} \int\left|\nabla \varphi_{1}\right|^{2} d \mu .
$$

Applying the COI in $B\left(x_{0}, 2 \kappa_{2} S\right)$ with $f=1$ and $s=2 \kappa_{2} S$ yields

$$
\int\left|\nabla \varphi_{1}\right|^{2} d \mu \leq c_{3} E\left(x_{0}, s\right)^{-1} \int_{B\left(x_{0}, 2 s\right)} d \mu=c_{3} V\left(x_{0}, 4 \kappa_{2} S\right) / E\left(x_{0}, 2 \kappa_{2} S\right) .
$$

Using (3.1) and (3.8) yields our result.

Combining with (6.2) tells us that

$$
\int_{B\left(x_{0}, R\right)}\left|w-w_{B\left(x_{0}, R\right)}\right|^{2} \gamma \leq c_{4} E\left(x_{0}, R\right) C\left(x_{0}, R\right)=c_{4} V\left(x_{0}, R\right) .
$$

Proof of Theorem 3.3. We proved that the EHI for $\mathcal{C}$ implies the API for $\mathcal{C}$ in Theorem 5.4.

That the EHI for $\mathcal{C}$ implies the COI for $\mathcal{C}$ is proved in almost the same way as in [3, Sect. 4]. We replace the use of $\psi(r)$ there by $E\left(x_{0}, r\right)$ and also replace appearances of $r^{\beta}$ by $E\left(x_{0}, r\right)$. The analogue of Lemma 4.7(a) of [3] follows from Proposition 4.1. To prove the analogue of [3, Lemma 4.7(b)], we use Proposition 5.1 and then follow the proof given in [3].

Away from the diagonal, the Green function is Hölder continuous in each variable by (3.12). The FVG condition of [3] is implied by our current volume growth condition.

With Propositions 6.1 and 6.2 in place of Propositions 5.2 and 5.7 of [3], we can follow the argument of [3, Section 5] to show that the API and COI together imply the EHI.

Proof of Theorem 2.8. If (2.1), Assumptions 2.1 and 2.2, and the EHI hold for $\left(\mathcal{G}, C_{x y}\right)$, Propositions 3.1 and 3.2 tell us that the corresponding facts hold for the cable system $\mathcal{C}$. By Theorem 3.3, the API and COI hold for $\mathcal{C}$, and by Proposition 3.2 again, the API and COI hold for the weighted graph. This proves (a). The proof of (b) is similar.

Proof of Theorem 2.9. Suppose (2.1) and Assumptions 2.1 and 2.2 hold for $\left(\mathcal{G}, C_{x y}\right)$ and for $\left(\mathcal{G}, C_{x y}^{\prime}\right)$. Suppose the EHI holds for $\left(\mathcal{G}, C_{x y}\right)$. Then by Theorem 2.8 the API and COI hold for $\left(\mathcal{G}, C_{x y}\right)$. Since $\left(\mathcal{G}, C_{x y}\right)$ and $\left(\mathcal{G}, C_{x y}^{\prime}\right)$ are equivalent weighted graphs, capacities of balls are comparable, and hence expected occupation times are comparable. Therefore the API and COI hold for $\left(\mathcal{G}, C_{x y}^{\prime}\right)$. By Theorem 2.8, the EHI holds for $\left(\mathcal{G}, C_{x y}^{\prime}\right)$.

\section{Further results}

\subsection{The general case}

We now consider the general case for infinite graphs. Theorem 7.1 can also be used in the transient case. 
For $x \in \mathcal{V}$ and $r \geq 1$, let $\widetilde{C}(x, r)$ be the capacity of $B(x, r)$ with respect to the process killed on exiting $B(x, 8 r)$. Thus

$$
\widetilde{C}(x, r)=\inf \left\{\mathcal{E}_{\mathcal{G}}(f, f):\left.f\right|_{B(x, r)}=1,\left.f\right|_{B(x, 8 r)^{c}}=0, f \in \mathcal{F}\right\} .
$$

Let $\widetilde{E}(x, r)=V(x, r) / \widetilde{C}(x, r)$. We assume (2.1), volume doubling, and expected occupation time growth (for $\widetilde{E})$. $\widetilde{C}(x, r)$ is no longer necessarily monotone in $r$, and so we must make an additional assumption, that of capacity comparability: there exists $c_{1}$ not depending on $x, y$, or $r$ such that if $d(x, y)<2 r$, then

$$
c_{1} \widetilde{C}(x, r) \leq \widetilde{C}(y, 2 r) \leq c_{1}^{-1} \widetilde{C}(x, r) .
$$

In particular, taking $x=y$ shows that $\widetilde{C}(x, r)$ and $\widetilde{C}(x, 2 r)$ are comparable. This implies expected occupation time comparability: there exists $c_{2}$ such that

$$
c_{2} \widetilde{E}(x, r) \leq \widetilde{E}(y, 2 r) \leq c_{2}^{-1} \widetilde{E}(x, r)
$$

Now define the API and COI in terms of $\widetilde{E}$ instead of $E$.

Theorem 7.1. Suppose that (2.1), volume doubling, expected occupation time growth, and capacity comparability hold for $\mathcal{G}$.

(a) If the EHI holds, then the API and COI hold.

(b) If the API and COI hold, then the EHI holds.

(c) Let $\left(\mathcal{G}, C_{x y}\right)$ and $\left(\mathcal{G}, C_{x y}^{\prime}\right)$ be equivalent graphs. Suppose (2.1), volume doubling, expected occupation time growth, and capacity comparability also hold for $\left(\mathcal{G}, C_{x y}^{\prime}\right)$. If the EHI holds for $\left(\mathcal{G}, C_{x y}\right)$, then it holds for $\left(\mathcal{G}, C_{x y}^{\prime}\right)$.

Proof. As in the proofs of Theorems 2.8 and 2.9, we immediately transfer to the cable system. The proof of Proposition 4.1 still applies and we find that $G_{B(x, r)}(x, y)$ is comparable to $1 / C(x, r)$. The proof of Proposition 4.2 shows that $\int_{B(x, r)} G_{B(x, 8 r)}(x, z) \mu(d z)$ is comparable to $\widetilde{E}(x, r)$.

For $x_{0} \in \mathcal{C}$ and $r>0$, let $D=B\left(x_{0}, 8 r\right)$. Then if $x, y \in B\left(x_{0}, r\right)$, we have

$$
\left(G_{D}\right)^{2}(x, y) \leq c_{1} \widetilde{E}\left(x_{0}, r\right) G_{D}(x, y)
$$

The proof of this is the same as the proof of Proposition 5.2, but we use (7.1) to compare $\widetilde{E}(x, r)$ and $\widetilde{E}(y, r)$. We then conclude

$$
G_{D}^{\alpha}(x, y) \geq c_{2} / \widetilde{C}\left(x_{0}, r\right)
$$

just as in the proof of Proposition 5.3. We then argue that the EHI implies the API as in the proof of Theorem 5.4. The remainder of the proof is as in Section 6. 


\subsection{Metric measure spaces}

There is no difficulty extending our theorems to more general continuous state spaces. See [4] for the definitions of all terms introduced in this subsection. Let $(X, d, \mu)$ be a metric measure space such that the metric is geodesic and $X$ has infinite diameter. Examples of such spaces include Riemannian manifolds, cable systems, Euclidean domains with smooth boundary, and fractals.

Let $(\mathcal{E}, \mathcal{F})$ be a local regular Dirichlet form. Associated to $f \in \mathcal{F} \cap L^{\infty}$ is a measure $\Gamma(f, f)(d x)$ characterized by

$$
\int_{X} \tilde{g}(x) \Gamma(f, f)(d x)=2 \mathcal{E}(f, f g)-\mathcal{E}\left(f^{2}, g\right)
$$

for all $g \in \mathcal{F} \cap L^{\infty}$, where $\widetilde{g}$ is the quasi-continuous modification of $g$. Define

$$
\mathcal{E}_{A}(f, f)=\int_{A} \Gamma(f, f)(d x)
$$

Let $B(x, r)$ be the ball of radius $r$, and $V(x, r)=\mu(B(x, r))$. Assume $(\mathcal{E}, \mathcal{F})$ is transient, let

$$
C(x, r)=\inf \left\{\mathcal{E}(f, f):\left.f\right|_{B(x, r)}=1, f \in \mathcal{F}\right\},
$$

and $E(x, r)=V(x, r) / C(x, r)$. Assume that Assumption 2.2 holds; the statement in the present context is the same as the one in Section 3 provided we drop the primes. Again dropping the primes, define the API, COI, and EHI as in Section 3. We need one more regularity condition, namely, that the associated continuous symmetric strong Markov process spends 0 time at any given point, or equivalently, for each $x$,

$$
G 1_{B(x, r)}(x) \rightarrow 0 \quad \text { as } r \rightarrow 0,
$$

where $G$ is the Green potential operator.

We then have the analogues of Theorems 2.8 and 2.9. We say two Dirichlet forms $\mathcal{E}$ and $\mathcal{E}^{\prime}$ are equivalent if they have the same domain $\mathcal{F}$ and there exists $c_{1}$ such that

$$
c_{1} \mathcal{E}(f, f) \leq \mathcal{E}^{\prime}(f, f) \leq c_{1}^{-1} \mathcal{E}(f, f), \quad f \in \mathcal{F} .
$$

Theorem 7.2. Assume that the analogues of Assumptions 2.1 and 2.2 hold, and (7.2) holds for $(\mathcal{E}, \mathcal{F})$.

(a) If the EHI holds, then the API and COI hold for $(\mathcal{E}, \mathcal{F})$.

(b) If the API and COI hold for $(\mathcal{E}, \mathcal{F})$, then the EHI holds for $(\mathcal{E}, \mathcal{F})$.

(c) Let $\mathcal{E}$ and $\mathcal{E}^{\prime}$ be equivalent. Assume that the analogues of Assumptions 2.1 and 2.2 hold and (7.2) holds for $\left(\mathcal{E}^{\prime}, \mathcal{F}\right)$. If the EHI holds for $\mathcal{E}$, then it holds for $\mathcal{E}^{\prime}$.

Proof. We modify the proof of Theorem 3.3 in a manner entirely similar to the way [4] extended the results of [3] to metric measure spaces. (7.2) comes in when deriving (4.1). The details are left to the interested reader.

Remark 7.3. We can similarly state and prove the analogue of Theorem 7.1.

Acknowledgments. I would like to thank Professors Martin Barlow, Moritz Kassmann, Takashi Kumagai, and Laurent Saloff-Coste for helpful discussions and comments. This research was partially supported by NSF grant DMS-0901505. 


\section{References}

[1] Barlow, M. T.: Diffusions on fractals. In: Lectures on Probability Theory and Statistics (SaintFlour, 1995), Springer, Berlin, 1-121 (1998) Zbl 0916.60069 MR 1668115

[2] Barlow, M. T., Bass, R. F.: Brownian motion and harmonic analysis on Sierpinski carpets. Canad. J. Math. 51, 673-744 (1999) Zbl 0945.60071 MR 1701339

[3] Barlow, M. T., Bass, R. F.: Stability of parabolic Harnack inequalities. Trans. Amer. Math. Soc. 356, 1501-1533 (2004) Zbl 1034.60070 MR 2034316

[4] Barlow, M. T., Bass, R. F., Kumagai, T.: Stability of parabolic Harnack inequalities on metric measure spaces. J. Math. Soc. Japan 58, 485-519 (2006) Zbl 1102.60064 MR 2228569

[5] Bass, R. F.: Probabilistic Techniques in Analysis. Springer, New York (1995) Zbl 0817.60001 MR 1329542

[6] Delmotte, T.: Graphs between the elliptic and parabolic Harnack inequalities. Potential Anal. 16, 151-168 (2002) Zbl 1081.39012 MR 1881595

[7] Fabes, E. B., Stroock, D. W.: A new proof of Moser's parabolic Harnack inequality via the old ideas of Nash. Arch. Mech. Ration. Anal. 96, 327-338(1986) Zbl 0652.35052 MR 0855753

[8] Fukushima, M., Oshima, Y., Takeda, M.: Dirichlet Forms and Symmetric Markov Processes. De Gruyter, Berlin (1994) Zbl 0838.31001 MR 1303354

[9] Grigor'yan, A. A.: The heat equation on noncompact Riemannian manifolds. Math. USSR-Sb. 72, 47-77 (1992) Zbl 0776.58035 MR 1098839

[10] Hebisch, W., Saloff-Coste, L.: On the relation between elliptic and parabolic Harnack inequalities. Ann. Inst. Fourier (Grenoble) 51, 1437-1481 (2001) Zbl 0988.58007 MR 1860672

[11] Kumagai, T., Sturm, K. T.: Construction of diffusion processes on fractals, $d$-sets, and general metric measure spaces. J. Math. Kyoto Univ. 45, 307-327 (2005) Zbl 1086.60052 MR 2161694

[12] Moser, J.: On Harnack's inequality for elliptic differential equations. Comm. Pure Appl. Math. 14, 577-591 (1961) Zbl 0111.09302 MR 0159138

[13] Moser, J.: A Harnack inequality for parabolic differential equations. Comm. Pure Appl. Math. 17, 101-134 (1964) Zbl 0149.06902 MR 0159139

[14] Saloff-Coste, L.: A note on Poincaré, Sobolev, and Harnack inequalities. Int. Math. Res. Notices 1992, no. 2, 27-38 Zbl 0769.58054 MR 1150597 